\title{
La fuerza isométrica del agarre en judo
}

\author{
Juan G. BONITCH-GÓNGORA* \& Filipa ALMEIDA \\ Departamento de Educación Física, Facultad de Ciencias del Deporte. Universidad de Granada \\ (España)
}

Recepción: 08/10/2013; Aceptación: 18/02/2014; Publicación: 21/02/2014.

REVISIÓN

\begin{abstract}
Resumen
Debido a que el agarre es un importante aspecto técnico-táctico a través del cual los judokas imponen su dominio sobre el adversario, dificultando que éste pueda realizar las técnicas de ataque oportunas y favoreciendo las propias, los judokas deben poseer altos niveles de fuerza isométrica y resistencia a este tipo de fuerza en la musculatura prensora de los antebrazos, como uno de los aspectos fundamentales para alcanzar el éxito. Este artículo revisa los perfiles de fuerza y resistencia muscular del agarre de judokas de diferentes sexos, edades y niveles competitivos. En general, el pico de fuerza isométrica de los judokas de élite no ha variado en los últimos 40 años y no parece diferenciarse del alcanzado por judokas de menor nivel competitivo o incluso del registrado en grandes grupos de población, lo que indica a los investigadores que la resistencia a la fuerza isométrica de prensión manual puede ser un parámetro más relevante en la evaluación de los judokas que el pico de fuerza isométrica, ya que en los combates deben mantener el agarre de manera continua durante periodos de tiempo relativamente largos y la fuerza máxima no se mantiene durante mucho tiempo. Sin embargo existen pocos estudios sobre la capacidad de resistir a sucesivas tensiones isométricas de prensión manual en judokas.
\end{abstract}

Palabras clave: Fuerza isométrica; agarre; kumikata; judo; rendimiento.

\section{Hand-grip isometric strength in judo}

\begin{abstract}
The grip is an important technical and tactical aspect through which the judokas dominate the adversary, hindering the application of appropriate techniques and favoring their own attack. The judokas must have high levels of isometric force and endurance to this type of force on the gripping muscles of the forearms, as one of the key aspects for success. This article reviews the grip muscular strength and endurance profiles of judokas of different groups (gender, age and competitive level). In general, the peak isometric strength of elite judokas has not changed in the last 40 years and is similar to that reached by non-elite judokas or even registered in large populations. This indicate that the evaluation of the isometric hand grip endurance may be a more relevant parameter than the peak isometric force in judokas, as during the bouts the grip must be maintained for relatively long periods of time and the maximum force cannot be maintained for long. However there are few studies on the ability to resist successive isometric handgrip stress in judokas.
\end{abstract}

Keywords: Isometric strength; grip; kumikata; judo; performance

\section{A força isométrica da preensão em judo}

\section{Resumo}

Dado que a preensão é um aspeto técnico-tático muito importante, através do qual os judocas impõem o seu domínio sobre o adversário, dificultando que a este possa realizar as técnicas de ataque oportunas e favorecendo as próprias, os judocas aplicam altos níveis de força isométrica e resistência a este tipo de força muscular. A preensão dos antebraços é um dos aspetos fundamentais para ter êxito. Este artigo apresenta uma revisão dos vários perfis de força e resistência muscular de preensão de judocas com diferentes idades, sexos e níveis competitivos. De uma forma geral, o pico da força isométrica dos judocas de elite não variou nos últimos 40 anos e não parece se diferenciar do alcançado por judocas de menor nível competitivo, incluindo o registado em grandes grupos de população. Neste sentido, os investigadores indicam que a resistência e a força isométrica de preensão manual podem ser parâmetros mais relevantes do que a avaliação do pico da força isométrica feita pelos judocas, já que nos combates estes devem manter a preensão de forma contínua e durante muito tempo. Todavia, ainda existem poucos estudos sobre a capacidade de resistir a sucessivas tensões isométricas de preensão manual em judocas.

Palavras-chave: Força isométrica; preensão; kumikata; judo; rendimento.

\footnotetext{
${ }^{*}$ E-mail: juanbonitch@ugr.es. Dirección: Departamento de Educación Física, Facultad de Ciencias del Deporte. Universidad
} de Granada. Carretera de Alfacar s/n, 18011, Granada (España). 


\section{1.- Introducción}

La fuerza del agarre es evaluada con frecuencia en diferentes ámbitos clínicos como un indicador del estado general de salud y del nivel de fuerza de los miembros superiores (Nicolay \& Walker, 2005; Schlüssel, Anjos, Vasconcellos, \& Kac, 2008; Vianna, Oliveira, \& Araújo, 2007). Su evaluación también se considera muy importante en el rendimiento de determinadas modalidades deportivas (Fry et al., 2006; Leyk et al., 2007; Visnapuu \& Jürimäe, 2007). El judo es un deporte de combate con agarre cuyo objetivo es proyectar al adversario sobre la espalda y/o controlarlo en el suelo. El primer contacto entre los dos judokas se realiza a través de las técnicas de agarre del judogi (traje de judo) del oponente, denominadas kumikata (Adams, 1992). El dominio del agarre se considera un factor determinante para el resultado del combate, ya que permite la ejecución de las técnicas de ataque y dificulta las acciones del adversario (Calmet, Miarka, \& Franchini, 2010; Farmosi, 1980; Franchini, Takito, Matheus, Brito-Vieira, \& Kiss, 1997; Little, 1991). El kumikata integra el enfoque táctico del combate, por tanto agarrar primero significa avanzar primero y estar más cerca de realizar una técnica de ataque, lo que obliga al adversario a adoptar un rol defensivo (Calmet, Miarka, \& Franchini, 2010). Por este motivo es importante aumentar la fuerza de prensión de las manos y las de tracción y empuje de la parte superior del cuerpo como uno de los factores determinantes del éxito (Silva, Fields, \& Heimsfields, 2011).

Varios autores han investigado el pico de fuerza isométrica del agarre en judokas (Ache Dias et al., 2012; Bonitch-Góngora, Bonitch-Domínguez, Padial, \& Feriche, 2012; Claessen et al., 1984; Franchini et al., 1997; Franchini, Takito, \& Bertuzzi, 2005a; Franchini, Takito, Kiss, \& Sterkowicz, 2005b; Farmosi, 1980; Little, 1991; Matsumoto et al., 1972). Son numerosos los factores que influyen en el resultado del pico de fuerza isométrica máxima en la prensión manual. Así, por ejemplo, la fuerza del agarre en la mano dominante alcanza su pico máximo entre los 18 y 31 años en hombres y entre los 31 y 59 años en mujeres, y desciende significativamente después de los 60 años de edad (Budziareck, Duarte, \& Barbosa-Silva, 2008), los hombres poseen mayores picos de fuerza y de potencia en el agarre que las mujeres (Aoki, \& Demura 2008; Dopsaj et al., 2007; Leyk et al., 2007), la masa corporal correlaciona positivamente con la fuerza del agarre (Coelho e Silva, Figueiredo, Carvalho, \& Malina, 2008), y los sujetos con mayor perímetro del antebrazo poseen mayores valores de fuerza en el agarre (Nicolay \& Walker, 2005). En esta línea, se ha encontrado una relación positiva entre la masa corporal y el pico de fuerza en la prensión manual en judokas (Thomas, Cox, LeGal, Verde, \& Smith, 1989), diferencias entre judokas de distintos grupos de edad (Little, 1991), y diferencias entre hombres y mujeres judokas en ambas manos (Franchini et al., 1997), aunque en esta investigación, cuando los datos se presentaban relativos a la masa corporal no se encontraron diferencias. Algunos estudios no encontraron diferencias en el pico de fuerza isométrica de prensión manual entre judokas y no judokas (Ache Dias et al., 2012) o entre judokas de distinto nivel competitivo (Franchini et al., 2005b; Gutiérrez-Sánchez, Soria-Domínguez, Pérez Turpin, Cortell Tormo, \& Suárez Llorca, 2011). Bonitch-Góngora, Almeida, Padial, BonitchDomínguez, y Feriche (2013), tampoco encontraron diferencias significativas entre judokas cadetes masculinos de élite y no-élite en el pico de fuerza isométrica de la mano dominante, pero sí entre las judokas de sexo femenino. Cuando los datos se expresaron relativos al área muscular del brazo en $\mathrm{N} / \mathrm{cm}^{2}$, sí se encontraron diferencias entre los judokas de élite y no-élite en ambos sexos.

Por otro lado, se hace difícil establecer una relación directa entre la mejora de la fuerza de los miembros superiores y el éxito en la competición (Banobic, 2001; Franchini et al., 2004), debido a la gran complejidad de las acciones técnicas y tácticas del combate, que constituyen un sistema de secuencias complejas y muy variadas en función de la situación y de las características del judoka. En ellas se incluyen diferentes fases como la adopción de una posición de "guardia", el intento de agarre del judogi del adversario, donde los judokas se desplazan por el tatami analizando la información, la elección del agarre más adecuado, la elección del tipo de ataque, directo o indirecto, etc. (Calmet, Miarka, \& Franchini, 2010). Además, el procedimiento de medida del pico de fuerza isométrica de la mano se ha realizado tradicionalmente mediante un test de una única contracción isométrica máxima de entre 5 y 15 s de duración. Sin embargo, este tipo de trabajo muscular no es el que se desarrolla durante el combate de judo, donde la duración de cada agarre oscila entre 11 y 21 s (Miarka, Julio, Del Vecchio, Calmet, \& Franchini, 2012) y se repite una media de 11 veces a lo largo del combate (Castarlenas \& Planas, 1997; Marcon, Franchini, Vieira, \& Barros, 2007) en los 8 min de media que duran los combates (Franchini, Del Vecchio, Matsushigue, \& Artioli, 2011), 
durante los cuales la mayor parte del tiempo del combate $(51 \pm 11 \%)$ se consume en la lucha por el agarre (Marcon, Franchini, Jardim, \& Neto, 2010), por lo que las exigencias de fuerza de la parte superior del cuerpo del judoka son extremadamente altas y están en demanda constante (BonitchDomínguez, Bonitch-Góngora, Padial, \& Feriche, 2010; Franchini, Nunes, Moraes, \& Del Vecchio, 2007; Miarka et al., 2012).

Así, la resistencia a la fuerza isométrica del agarre podría ser un factor más condicionante del éxito en judo que el pico de fuerza. En este sentido, Ache Dias et al. (2012) no encontraron diferencias en el pico de fuerza entre judokas y no judokas, pero los judokas eran más resistentes a la fatiga. Dos estudios encuentran pérdidas del 39\% (Bonitch-Góngora et al., 2007) y de entre el 24,5\% y el 18,8\% (Bonitch-Góngora et al., 2013), después de, respectivamente, 10 y 8 series de $10 \mathrm{~s}$ de contracción isométrica máxima con $10 \mathrm{~s}$ de descanso pasivo en judokas masculinos de diferentes niveles competitivos, mostrándose más resistentes a la fatiga aquellos judokas de más nivel. Otra investigación (Franchini et al., 2011), no encuentran diferencias en la resistencia al agarre isométrico en un test de suspensión en judogi con brazos extendidos entre judocas de nivel nacional y regional, pero sí cuando el test de resistencia era dinámico (medido por las repeticiones de tracciones en la barra con agarre isométrico manual en judogi). Sin embargo, Aruga, Nakanishi, Yamashita, Onda, y Ubukata (2006) no encontraron diferencias en el mismo test entre judokas japoneses de diferente nivel competitivo. Por otro lado, el número de estudios que abordan el efecto del combate sobre la fuerza isométrica del agarre en judokas es muy limitado. Los estudios de Bonitch-Góngora et al. (2012) y de Iglesias, Clavel, Dopico, \& Tuimil (2003), reportaron pérdidas del $15 \%$ tras cuatro y dos combates de judo respectivamente.

Es bien sabido que la comprensión de las características de los atletas de élite puede proporcionar información detallada con respecto a lo que se necesita para el alcanzar el éxito competitivo. Por ello, el objetivo de la presente revisión bibliográfica es presentar y discutir el conocimiento actualmente disponible sobre la problemática del agarre en judo y los factores de los que depende.

\section{2.- Método}

En esta revisión se han incluido los estudios que investigaron las características del pico de fuerza isométrica (una contracción < $10 \mathrm{~s}$ ) y/o la capacidad de resistir a prolongadas y/o sucesivas contracciones isométricas del agarre, en judokas y no judokas o entre judokas de diferentes niveles competitivos. Estos estudios fueron seleccionados en función de su objetivo, metodología, y el número y las características de los judokas evaluados, y atendiendo a su disponibilidad las consideraciones específicas sobre las diferencias entre los grupos de judokas son relativas a la edad, el sexo, el peso corporal y el nivel competitivo. Para la localización de los estudios se realizó una búsqueda bibliográfica en PubMed, SportDiscus e ISI Web of Knowledge mediante las palabras clave específicas "judo and isometric strength", "judo and grip", "judo and kumi-kata", "judo and strength", "judo and force" y "judo and physical fitness". Posteriormente se recogieron más estudios tras el examen de las citas presentes dentro de las publicaciones recogidas, que de no encontrarse en las citadas bases de datos, se buscaron en los buscadores Google y Google Scholar. Finalmente se incluyeron 19 artículos, 13 de los cuales describían el valor pico de fuerza isométrica, a nivel absoluto y/o relativo, y 6 que analizaban la resistencia a la fuerza isométrica del agarre en diferentes tareas, con un total de 728 sujetos participantes, divididos en 37 grupos.

\section{3.- Fuerza isométrica máxima del agarre}

Algunas investigaciones no encontraron diferencias en el pico de fuerza isométrica del agarre entre judokas de distinto nivel competitivo (Bonitch-Góngora et al., 2013; Franchini et al., 2005b; Gutiérrez-Sánchez et al., 2011). Por otro lado, los valores absolutos de los judokas masculinos se muestran similares en judokas cadetes (Franchini, Takito, \& Kiss, 2000), júnior y séniors brasileños no medallistas nacionales (Franchini et al., 2005a), judokas universitarios españoles (Cortell-Tormo et al., 2013) y cadetes canadienses de nivel regional (Little, 1991) (Tabla 1). 
Tabla 1

Pico de fuerza isométrica del agarre en judokas ( $\mathrm{kg})$.

\begin{tabular}{|c|c|c|c|}
\hline Estudio & Características de los judokas; sexo & $\begin{array}{l}\text { FI derecha } \\
(\mathrm{M} \pm \mathrm{DT})\end{array}$ & $\begin{array}{l}\text { FI izquierda } \\
(\mathrm{M} \pm \mathrm{DT})\end{array}$ \\
\hline $\begin{array}{l}\text { Matsumoto et al. } \\
\qquad(1972)\end{array}$ & $\begin{array}{l}\text { Judokas japoneses universitarios }(\sim 66 \mathrm{~kg}) ; \text { Masc } \\
(\mathrm{n}=12) \text { : } \\
1967 \\
1968 \\
\text { Participantes en el Cto. Mundial Universitario } 1967 \\
(\sim 73 \mathrm{~kg} ; \mathrm{n}=18) \text { : } \\
1967 \\
1968 \\
\text { Judokas universitarios }(\sim 83 \mathrm{~kg} ; \mathrm{n}=8) \text { : } \\
1967 \\
1968\end{array}$ & $\begin{array}{l}43.8 \\
49.3 \\
44.9 \\
\\
50.8 \\
53.3 \\
\\
55.3 \\
59.6\end{array}$ & $\begin{array}{l}43.8 \\
49.3 \\
45.1 \\
47.7 \\
52.2 \\
49.5 \\
55.6\end{array}$ \\
\hline Farmosi (1980) & $\begin{array}{l}\text { Equipo nacional húngaro; } \\
\text { Masc: Todos }(\mathrm{n}=18) \\
<71 \mathrm{~kg}(\mathrm{n}=7) \\
>71 \mathrm{~kg}(\mathrm{n}=11)\end{array}$ & $\begin{array}{c}59.9 \pm 11.2 \\
54.3 \pm 5.4 \\
63.9 \pm 12.8\end{array}$ & $\begin{array}{c}55.7 \pm 10.7 \\
50.9 \pm 5.4 \\
59.0 \pm 12.4 \\
\end{array}$ \\
\hline $\begin{array}{l}\text { Claessens et al. } \\
\qquad(1984)\end{array}$ & $\begin{array}{l}\text { Judokas belgas de alto nivel: } \\
\text { Todos }(\mathrm{n}=24) \\
<71 \mathrm{~kg}(\mathrm{n}=13) \\
71-86 \mathrm{~kg}(\mathrm{n}=9)\end{array}$ & $\begin{array}{l}64.9 \pm 8.9 \\
56.8 \pm 7.7 \\
59.7 \pm 6.1\end{array}$ & $\begin{array}{l}59.7 \pm 8.8 \\
54.4 \pm 7.5 \\
59.3 \pm 7.6\end{array}$ \\
\hline $\begin{array}{l}\text { Thomas et al. } \\
\text { (1989) }\end{array}$ & $\begin{array}{l}\text { Equipo nacional canadiense (1987); Masc: } \\
\text { Todos }(n=22)\end{array}$ & $56.4 \pm 6.6$ & $55.7 \pm 6.6$ \\
\hline Little (1991) & $\begin{array}{l}\text { Judokas canadienses: } \\
\text { Junior Fem }(n=9) \\
\text { Senior Fem }(n=8) \\
\text { Cadete Masc }(n=17) \\
\text { Junior Masc }(n=9) \\
\text { Senior Masc }(n=17)\end{array}$ & $\begin{array}{c}32.1 \pm 3.5 \\
31.8 \pm 5.8 \\
39.8 \pm 12.7 \\
52.0 \pm 8.3 \\
57.7 \pm 9.0 \\
\end{array}$ & $\begin{array}{c}29.3 \pm 5.3 \\
30.6 \pm 5.4 \\
39.4 \pm 10.0 \\
50.6 \pm 8.5 \\
54.0 \pm 10.4\end{array}$ \\
\hline $\begin{array}{l}\text { Franchini et } \\
\text { al.(1997) }\end{array}$ & $\begin{array}{l}\text { Equipo brasileño universitario (1996): } \\
\text { Masc }(n=6) \\
\text { Fem }(n=7)\end{array}$ & $\begin{array}{l}49.5 \pm 12.8 \\
32.3 \pm 7.6\end{array}$ & $\begin{array}{l}47.2 \pm 12.4 \\
32.2 \pm 7.8\end{array}$ \\
\hline $\begin{array}{l}\text { Franchini et al. } \\
\qquad(2005 a)\end{array}$ & Equipo brasileño univ.; Masc: Todos $(n=13)$ & $54.3 \pm 8.3$ & $53.2 \pm 7.4$ \\
\hline $\begin{array}{l}\text { Franchini et al. } \\
\qquad(2005 b)\end{array}$ & $\begin{array}{l}\text { Judokas brasileños; Masc: } \\
\text { Elite }(\mathrm{n}=26) \\
\text { No-elite }(\mathrm{n}=66)\end{array}$ & $\begin{array}{l}51.0 \pm 10.0 \\
42.0 \pm 11.0\end{array}$ & $\begin{array}{l}49.0 \pm 10.0 \\
40.0 \pm 10.0\end{array}$ \\
\hline $\begin{array}{l}\text { Gutiérrez-Sánchez } \\
\text { et al. (2011) }\end{array}$ & $\begin{array}{l}\text { Judokas participantes en el Cto. de Galicia Sub-20: } \\
\text { Todos }(n=102)\end{array}$ & $\begin{array}{c}\text { Dominante } \\
44.8 \pm 9.2\end{array}$ & $\begin{array}{c}\text { No-Dominante } \\
421 \pm 90.56\end{array}$ \\
\hline $\begin{array}{l}\text { Ache-Días et al. } \\
\text { (2012) }\end{array}$ & $\begin{array}{l}\text { Judokas brasileños sénior de nivel nacional; Masc: } \\
\text { Todos }(\mathrm{n}=22)\end{array}$ & $\begin{array}{l}\text { Dominante } \\
52.4 \pm 8.3\end{array}$ & $\begin{array}{l}\text { No-Dominante } \\
52.4 \pm 8.0\end{array}$ \\
\hline $\begin{array}{l}\text { Bonitch-Góngora } \\
\text { et al. (2012) }\end{array}$ & $\begin{array}{l}\text { Judokas Sub-20 y Sub-23 medallistas nacionales en } \\
\text { España. Todos }(n=12)\end{array}$ & $54.6 \pm 7.9$ & $52.9 \pm 7.9$ \\
\hline $\begin{array}{l}\text { Bonitch-Góngora } \\
\text { et al. (2013) }\end{array}$ & $\begin{array}{l}\text { Élite (Equipos Nacionales Cadetes de Dinamarca, } \\
\text { Portugal y Suecia) } \\
\text { Masc }(n=26) \\
\text { Fem }(n=21) \\
\text { No-élite (Equipo Regional de Andalucía) } \\
\text { Masc }(n=19) \\
\text { Fem }(n=7)\end{array}$ & $\begin{array}{l}\text { Dominante } \\
47.0 \pm 9.4 \\
32.5 \pm 4.1 \\
42.3 \pm 7.2 \\
23.6 \pm 6.5\end{array}$ & \\
\hline $\begin{array}{l}\text { Cortell-Tormo et } \\
\text { al. (2013) }\end{array}$ & $\begin{array}{l}\text { Judokas participantes en el Cto. Nacional Universitario } \\
\text { de España. Todos }(n=54): \\
50-66 \mathrm{Kg}(\mathrm{n}=15) \\
67-81(\mathrm{n}=21) \\
+81(\mathrm{n}=18)\end{array}$ & $\begin{array}{l}44.85 \pm 6.63 \\
50.12 \pm 7.87 \\
54.15 \pm 7.16 \\
\end{array}$ & \\
\hline
\end{tabular}

Abreviaturas: $\mathrm{M}=$ media aritmética, DT=desviación típica, Masc: masculino, Fem: femenino 
En la comparación entre géneros, los datos indican que los judokas masculinos sénior (Claessens et al., 1984; Franchini et al., 2005b) desarrollan más pico de fuerza isométrica que las judokas femeninas sénior (Leyk et al., 2007; Little, 1991), lo que está de acuerdo con otros estudios sobre la influencia del género en la capacidad de desarrollar fuerza en la mano (Aoki \& Demura 2008; Dopsaj et al., 2007). Según algunos autores, esto puede deberse a la mayor masa muscular de los hombres (Imrhan, 2003; Nevill \& Holder, 2000; Peebles \& Norris 2003; Peolsson, Hedlund, \& Oberg, 2001; Ruiz-Ruiz, Mesa, Gutiérrez, \& Castillo, 2002), sobre todo en la parte superior del cuerpo (Miller, Macdougall, Tarnopolsky, \& Sale, 1993). Esto se ve corroborado por Franchini et al., (1997) en su estudio realizado a judokas de la selección brasileña universitaria de judo, donde encontraron diferencias significativas en la fuerza isométrica máxima de la mano entre hombres y mujeres. Estos datos deben tratarse con precaución, ya que en algunos de estos estudios no se estratificó la muestra según categorías de peso. En este sentido, varias investigaciones han encontrado una correlación positiva entre la fuerza isométrica máxima de la mano y el peso corporal (Budziareck et al., 2008; Coelho e Silva et al., 2008; Thomas et al., 1989). Franchini et al., (2011), en una reciente revisión sobre los perfiles fisiológicos de los judokas, puntualizan que en la investigación de Matsumoto et al., (1972), a pesar de que los autores no establecen ninguna comparación estadística entre los diferentes grupos de peso, parece que la fuerza isométrica aumenta de acuerdo con la categoría de peso.

Son escasas las investigaciones que aportan valores relativos de fuerza isométrica de la prensión manual en judokas, comparando la muestra en cuanto a la edad, el nivel competitivo y/o el sexo. Así, Little (1991) encontró diferencias en la fuerza isométrica máxima del agarre entre judokas cadetes y junior y sénior, sin embargo, cuando un índice de la fuerza isométrica (incluyendo el agarre, la espalda y el brazo) se expresó en relación a la masa corporal, solamente los judokas cadetes y sénior presentaron diferencias entre ellos. Franchini et al. (2005b) no encontraron diferencias en la fuerza isométrica manual entre judokas de élite y no-élite júnior y sénior, utilizando la masa corporal como co-variable, lo que es un procedimiento similar a la relativización. Un estudio (Franchini et al., 1997) presentó mayores valores absolutos de fuerza isométrica del agarre en ambas manos de los judokas masculinos respecto a las judokas femeninas. Sin embargo cuando los valores se presentaron relativos a la masa corporal, no se observaron diferencias. Franchini et al, (2011) establecen la hipótesis de que la diferencia en la fuerza isométrica del agarre es menor entre hombres y mujeres de alto nivel que entre hombres y mujeres de menor nivel competitivo, probablemente porque el entrenamiento de alta intensidad reduce las diferencias en la fuerza relativa. En este sentido, el hecho de que Bonitch-Góngora et al. (2013), publicaran diferencias en el pico de fuerza isométrica del agarre entre judokas de élite y no-élite cadetes en ambos sexos cuando los datos se expresaron relativos al área muscular del brazo $\left(\mathrm{N} / \mathrm{cm}^{2}\right)$, puede obedecer a esta explicación, ya que a estas edades aún las intensidades de entrenamiento son bajas.

Por otro lado, algunos autores (Anakwe, Huntley, \& Mceachan, 2007; Nicolay \& Walker 2005) afirman que las medidas antropométricas del antebrazo son un mejor predictor de la fuerza del agarre que la masa corporal y la altura, debido a que muestran correlaciones más fuertes con el pico de fuerza. Bonitch-Góngora et al. (2013), encontraron altas correlaciones entre la circunferencia del antebrazo $(\mathrm{cm})$ y el área muscular del brazo $\left(\mathrm{N} / \mathrm{cm}^{2}\right)(\mathrm{r}=0.79, \mathrm{p} \leq 0.01)$, y el pico de fuerza isométrica $(\mathrm{r}=0.80, \mathrm{p} \leq 0.01)$. Teniendo en cuenta estas evidencias, una importante conclusión es que presentar los valores relativos a las medidas antropométricas del miembro superior, como la circunferencia del antebrazo o el área muscular del brazo, nos permite diferenciar con mayor precisión a judokas de elite y de no-elite en lo que a la fuerza isométrica máxima del agarre se refiere.

\section{4.- Resistencia a la fuerza isométrica del agarre}

Dempsey y Ayoub (1996) concluyeron que la fuerza que puede mantenerse en el agarre es menor que la fuerza que puede alcanzarse inicialmente. Por otro lado, Nicolay y Walker (2005) afirman que la capacidad de ejercer fuerza isométrica máxima en la mano decrece a medida que la duración de la contracción aumenta, y Walker, Siddiqi, y Amundsen (2002) sugieren que el porcentaje de pérdida de fuerza isométrica del agarre podría estar relacionado con el valor inicial, 
lo que podría traducirse en una mayor pérdida en aquellos sujetos que desarrollan mayor pico de fuerza isométrica inicial. En esta línea, Bonitch-Góngora et al. (2007), en un protocolo de 10 series de $10 \mathrm{~s}$ de contracción isométrica máxima, separados por $10 \mathrm{~s}$ de descanso pasivo, encontraron pérdidas del $39 \%$ respecto a la fuerza isométrica máxima de la mano dominante $(\mathrm{p}<0.01)$ en judokas austríacos sénior de nivel nacional e internacional. El mayor porcentaje de pérdida registrado en los judokas de más nivel competitivo, que desarrollaron mayores niveles de fuerza isométrica máxima de partida, apoya esta hipótesis. Sin embargo, aunque las pérdidas de fuerza isométrica son superiores en el grupo de judokas de mayor nivel frente al grupo de judokas de menor nivel, los primeros desarrollaron siempre niveles más altos de fuerza isométrica máxima relativa en cada una de las repeticiones efectuadas. Estos resultados son corroborados en el estudio de Bonitch-Góngora et al. (2013) con judokas cadetes, donde con el mismo protocolo, pero esta vez con 8 series, obtienen pérdidas (en la mano dominante) del 24,5 $\pm 9,1 \%$ y 18,8 $\pm 9,1 \%$ en hombres y del $18,4 \pm 9,3 \%$ y $16,8 \pm 7,0 \%$ en mujeres, élite y no-élite respectivamente, entre la $1^{\mathrm{a}}$ y la $8^{\mathrm{a}}$ serie. Igual que ocurre con los judokas sénior, las pérdidas son superiores en aquellos sujetos que desarrollan más pico inicial de fuerza isométrica del agarre, aunque, por otro lado, consiguen mantener un pico más elevado durante todas las series. Estos hallazgos permiten demostrar por primera vez que los judokas de más nivel podrían aplicar mayores niveles de fuerza en el agarre durante todo el combate que aquellos judokas de menor nivel competitivo, imponiéndose en la lucha por el kumikata y aumentando así las posibilidades de éxito sobre sus rivales.

Dos investigaciones midieron la resistencia isométrica de prensión manual a través de diferentes tests de suspensión con agarre sobre un judogi (Tabla 2). Franchini et al. (2011), no encuentran diferencias en la resistencia al agarre isométrico en un test de suspensión en judogi con brazos extendidos entre judocas de nivel nacional y regional, pero sí cuando el test de resistencia era dinámico (medido por las repeticiones de tracciones en la barra con agarre isométrico manual en judogi). Sin embargo, Aruga et al. (2006) no encontraron diferencias en este último test entre judokas japoneses de diferente nivel competitivo. Así, es posible que la resistencia de fuerza isométrica diferencie a judokas y no judokas, que la resistencia de fuerza dinámica con ejecución de la prensión manual isométrica diferencie a judokas de diferentes niveles, pero que después de un cierto grado de desarrollo, no se diferencie más a judokas de diferentes niveles en cuanto a resistencia de prensión manual o dinámica, por ejemplo, pudiendo ser que haya un mínimo necesario a desarrollar.

Solamente dos estudios han investigado el efecto de la sucesión de varios combates de judo en la fuerza isométrica de la mano (Tabla 2). Iglesias et al. (2003) simularon una competición compuesta por dos combates, separados por $15 \mathrm{~min}$ de descanso pasivo, donde encontraron pérdidas de fuerza isométrica en ambas manos del $5 \%$ después del primer combate, y del 15\% en el segundo, en judokas de categoría Sub-23 y séniors de nivel nacional. Bonitch-Góngora et al., (2012), con el mismo protocolo realizaron cuatro combates y encontraron pérdidas del $12.7 \%$ después del primer combate, y del $15.0 \%$ después de los cuatro combates, en la fuerza de prensión de la mano dominante en judokas Sub-23 y séniors de nivel nacional. Este estudio halló diferencias significativas entre todas las medidas de fuerza isométrica del agarre tomadas antes de los combates, pero no entre todas las de después. El límite de máxima pérdida se mantuvo estable durante los cuatro combates y se relacionó con la concentración de ácido láctico post esfuerzo, en particular en la mano dominante. Esto demuestra que judokas no son capaces de recuperar sus niveles iniciales de fuerza del agarre durante todo el curso del torneo y, por primera vez, sugiere que la fatiga de cada mano depende de diferentes factores. Estas pérdidas son inferiores a las registradas por Bonitch-Góngora et al., (2007), en el estudio descrito más arriba, lo que contribuye a demostrar que durante los combates se aplican valores submáximos de fuerza isométrica en el agarre, mientras que el estudio de Bonitch-Góngora et al., (2007), se aplican valores máximos en cada una de las repeticiones. Esto está de acuerdo con los resultados de Yamaji, Demura, Nagasawa, y Nakada (2006), que afirman que la tasa de pérdida de fuerza de prensión manual aumenta cuando se realiza a una intensidad del $100 \%$ de la fuerza isométrica máxima.

Varios autores han investigado las causas de la fatiga originada por contracciones isométricas del agarre a diferentes intensidades. A intensidades superiores al 90-95\% de la fuerza isométrica máxima mantenidas por cualquier periodo de tiempo, la concentración de adenosintrifosfato y fosfocreatina en el músculo cae a menos del 30\% (Bangsbo, Johansen, 
Quistorff, \& Saltin, 1993) y la contribución del metabolismo anaeróbico láctico disminuye (Sahlin, 1978) debido a la naturaleza de su relación intensidad-tiempo. Por el contrario, cuando la contracción es menos intensa, lo que permite aumentos periódicos en el flujo sanguíneo al músculo, la concentración de lactato aumenta (120 mmol $\cdot \mathrm{kg}^{-1}$ de músculo seco al $25-60 \%$ vs $90-95 \%$ de la fuerza isométrica máxima) (Taylor \& Brassard, 1981) y su contribución a la fatiga periférica durante el agarre es más probable (Byström, \& Kilbom 1990; Karlsson, Bonde-Petersen, Henriksson, \& Knuttgen, 1975).

Tabla 2

Resistencia a la fuerza isométrica del agarre en judokas.

\begin{tabular}{|c|c|c|}
\hline Estudio & Características de los sujetos y el diseño & Resultados \\
\hline Iglesias et al. (2003) & $\begin{array}{l}\text { Judokas españoles masculinos Sub-23 y séniors } \\
\text { de nivel nacional; Todos }(n=6) \\
2 \text { combates de judo con } 15 \text { min de descanso } \\
\text { pasivo entre cada combate }\end{array}$ & $\begin{array}{l}\text { Pérdidas del } 5 \% \text { después del } \\
\text { primer combate, y del } 15 \% \\
\text { después del segundo en la mano } \\
\text { dominante }\end{array}$ \\
\hline Aruga et al. (2006) & $\begin{array}{l}\text { Judokas japoneses universitarios } \\
\text { Test de máximo } \mathrm{n} \text { o de flexiones de brazos } \\
\text { (dominadas) con agarre sobre judogi. }\end{array}$ & $\begin{array}{l}\text { No existió una correlación } \\
\text { positiva entre el no }{ }^{\circ} \text { de } \\
\text { repeticiones en el test de } \\
\text { flexiones de brazos con agarre } \\
\text { sobre judogi y el nivel } \\
\text { competitivo de los judokas } \\
\text { (ranking y resultados) }\end{array}$ \\
\hline $\begin{array}{l}\text { Bonitch-Góngora et } \\
\text { al. (2007) }\end{array}$ & $\begin{array}{l}\text { Equipo nacional sénior de Austria; Todos }(n=15) \\
10 \text { series de } 10 \text { s de contracción isométrica } \\
\text { máxima, con } 10 \text { s de descanso pasivo }\end{array}$ & $\begin{array}{l}\text { Pérdidas del } 39 \% \text { de la fuerza } \\
\text { isométrica máxima de la mano } \\
\text { dominante, entre la } 1^{\mathrm{a}} \text { y } 10^{\mathrm{a}} \text { serie }\end{array}$ \\
\hline $\begin{array}{l}\text { Franchini et al. } \\
\text { (2011) }\end{array}$ & $\begin{array}{l}\text { Élite (Equipo Nacional Sénior de Brasil): } \\
\text { Masc ( } n=16 \text { ) } \\
\text { No-élite (Equipo Regional de Barueri): } \\
\text { Masc ( } n=12 \text { ) } \\
\text { Test de máximo tiempo de suspensión con agarre } \\
\text { sobre judogi y brazos extendidos } \\
\text { Test de máximo no de flexiones de brazos } \\
\text { (dominadas) con agarre sobre judogi. }\end{array}$ & $\begin{array}{l}\text { Los judokas de élite y no-élite no } \\
\text { difirieron entre sí en el test de } \\
\text { máximo tiempo en suspensión } \\
\text { con agarre en judogi. Sin } \\
\text { embargo, los judokas de élite } \\
\text { ejecutaron un mayor } \mathrm{n}^{\circ} \text { de } \\
\text { repeticiones en el test de } \\
\text { flexiones de brazos con agarre } \\
\text { sobre judogi }(p<0,05)\end{array}$ \\
\hline $\begin{array}{l}\text { Bonitch-Góngora et } \\
\text { al. (2012) }\end{array}$ & $\begin{array}{l}\text { Judokas españoles masculinos Sub- } 20 \text { y Sub- } 23 \\
\text { de nivel nacional; Todos }(n=12) \\
4 \text { combates de judo con } 15 \text { min de descanso } \\
\text { pasivo entre cada combate }\end{array}$ & $\begin{array}{l}\text { Pérdidas del } 12,7 \% \text { después del } \\
\text { primer combate, y del } 15 \% \\
\text { después de los cuatro combates, } \\
\text { en la mano dominante }\end{array}$ \\
\hline $\begin{array}{l}\text { Bonitch-Góngora et } \\
\text { al. (2013) }\end{array}$ & $\begin{array}{l}\text { Élite (Equipos Nacionales Cadetes de Dinamarca, } \\
\text { Portugal y Suecia): } \\
\text { Masc }(n=26) \\
\text { Fem }(n=21) \\
\text { No-élite (Equipo Regional de Andalucía): } \\
\text { Masc ( } n=19) \\
\text { Fem ( } n=7) \\
8 \text { series de } 10 \text { s de contracción isométrica } \\
\text { máxima, con } 10 \text { s de descanso pasivo entre cada } \\
\text { serie }\end{array}$ & $\begin{array}{l}\text { Pérdidas (en la mano dominante) } \\
\text { del } 24,5 \pm 9,1 \% \text { y } 18,8 \pm 9,1 \% \text { en } \\
\text { hombres y del } 18,4 \pm 9,3 \% \text { y } 16,8 \\
\pm 7,0 \% \text { en mujeres, élite y no-élite } \\
\text { respectivamente, entre la } 1^{\text {a }} \text { y la } \\
8^{-} \text {serie }\end{array}$ \\
\hline
\end{tabular}

Abreviaturas: M=media aritmética, DT=desviación típica, Masc: masculino, Fem: femenino

\section{5.- Conclusiones}

Se ha demostrado la fatigabilidad de la musculatura prensora de los antebrazos durante los combates de judo (Bonitch-Góngora et al., 2012), lo que ha contribuido a definir la dinámica de la fuerza del agarre y las razones de fatiga. Así, las necesidades el agarre en judo parecen responder a un perfil de resistencia de fuerza que parece depender de factores relacionados con la fatiga muscular periférica, como la acidosis metabólica (Bonitch-Góngora et al., 2012). Otros estudios 
vinculan directamente el papel de la resistencia a la fuerza isométrica máxima del agarre con el resultado de la competición, siendo los judokas de más nivel capaces de resistir mejor a tareas de resistencia a la fuerza isométrica del agarre (Bonitch-Góngora et al., 2013; Franchini et al., 2011). Sin embargo, a pesar de que los datos analizados ponen de manifiesto que no existen diferencias en el pico de fuerza isométrica del agarre entre judokas de diferentes niveles competitivos (BonitchGóngora et al., 2013; Franchini et al., 2005b; Gutiérrez-Sánchez et al., 2011) o entre judokas y no judokas (Ache Dias et al., 2012), se ha puesto de manifiesto que un pico de fuerza isométrica más elevado permitirá a los judokas mantener niveles más altos durante el transcurso de una serie de contracciones isométricas máximas (Bonitch-Góngora et al., 2013).

Por tanto, teniendo en cuenta que el mantenimiento de la calidad del agarre durante un combate de judo es un factor determinante del éxito (Adams, 1992; Calmet, et al., 2010), la literatura revisada sugiere que puede depender tanto del pico como de la resistencia a la fuerza isométrica del agarre. Sin embargo, el hecho de que los judokas de alto nivel no se diferencien de judokas de menos nivel o de no judokas en cuanto al pico de fuerza isométrica del agarre, hace pensar que la capacidad de resistir a sucesivas contracciones sea un factor más decisivo durante los combates. Estos hallazgos proporcionan una valiosa información a los entrenadores para diseñar los programas de entrenamiento y mejorar el rendimiento. Se recomienda el diseño de regímenes de entrenamiento para aumentar el pico de fuerza isométrica y especialmente la fuerza-resistencia de la musculatura prensora de los antebrazos.

\section{Nota}

Una versión previa de este trabajo se presentó en el I Congreso Internacional de Judo De FrutosUMH, Alicante, 27-30 de junio de 2013.

\section{Referencias}

Ache Dias, J., Wentz, M., Külkamp, W., Mattos, D., Goethel, M., \& Borges Júnior, N., (2012). Is the handgrip strength performance better in judokas than in non-judokas?. Science \& Sports, 27, e9-e14.

Adams, N. (1992). Los agarres. Barcelona: Paidotribo.

Anakwe, R.E., Huntley, J.S., \& Mceachan, J.E. (2007). Grip strength and forearm circumference in a healthy population. Journal of Hand Surgery, 32, 203-209.

Aoki, H., \& Demura, S. (2008). The effect of gender and lateral dominance on gripping muscle power in young adults. Sport Sciences for Health, 3, 1-6.

Aruga, S., Nakanishi, H., Yamashita, Y., Onda, T., \& Ubukata, K. (2006). A Study on the training method for improving judo players' kumite strength - on the judogi chin-up method. Tokai Journal of Sports Medical Science Research Institute of Sports Medical Science, 18(1), 44-53.

Bangsbo, J., Johansen, L., Quistorff, B., \& Saltin, B. (1993). NMR and analytic biochemical evaluation of $\mathrm{CrP}$ and nucleotides in the human calf during muscle contraction. Journal of Applied Physiology, 74, 2034-2039.

Banobic, I. (2001). Posible judo performance prediction based on certain motor abilities and technical knowledge (skills) assessment. Kinesiology, 33(2), 191-206.

Bonitch-Domínguez, J., Bonitch-Góngora, J., Padial, P., \& Feriche, B. (2010). Changes in the peak legpower induced by successive judo bouts and their relationship to lactate production. Journal of Sports Sciences, 28, 1527-1534.

Bonitch-Góngora, J., Bonitch-Domínguez, J., Feriche, B., Chirosa, I., Sánchez, C., Granados, M.A., García, Z., Pintor, M.A, \& Padial, P. (2007). Análisis del comportamiento de la resistencia a la fuerza isométrica máxima de la musculatura prensora del antebrazo en judokas. Archivos de Medicina, 24(121), 358.

Bonitch-Góngora, J., Bonitch-Domínguez, J., Padial, P., \& Feriche, B. (2012). The effect of lactate concentration on the handgrip strength during judo bouts. Journal of Strength and Conditional Research, 26(7), 1863-1871. 
Bonitch-Góngora, J., Almeida, F., Padial, P., Bonitch-Domínguez, J., \& Feriche, B. (2013). Maximal isometric handgrip strength and endurance differences between elite and non-elite young judo athletes. Archives of Budo, 26(7), 239-248.

Budziareck, M.B., Duarte, R.R., \& Barbosa-Silva, M.C. (2008). Reference values and determinants for handgrip strength in healthy subjects. Clinical Nutrition, 27, 357-362.

Byström, S.E.G., \& Kilbom, A. (1990). Physiological response in the forearm during and after isometric intermittent handgrip. European Journal of Applied Physiology, 60, 457-466.

Calmet, M., Miarka, B., \& Franchini, E. (2010). Modeling of grasps in judo contests. International Journal of Performance Analysis in Sport, 10, 229-240.

Claessens, A.L., Beunen, G.P., Simons, J.M., Wellens, R.I., Gelfold, D., Nuyts, M.M. (1984). Body structure, somatotype and motor fitness of top class Belgian judoists. En: Day JA (ed.), The 1984 Olympic Scientific Congress Proceedings: perspectives in kinanthropometry (pp. 155163). Champaign, Il: Human Kinetics.

Castarlenas, J.L. \& Planas, A. (1997). Estudio de la estructura temporal del combate de judo. Apunts Edución Física y Deportes, 47, 32-9.

Coelho e Silva, M.J., Figueiredo, A.J., Carvalho, H.M., \& Malina, R.M. (2008). Functional capacities and sport-specific skills of 14- to 15-year-old male basketball players: size and maturity effects. European Journal of Sport Scicience, 8(5), 277-285.

Cortell-Tormo, J.M., Perez-Turpin, J.A., Lucas-Cuevas, A.G., Perez-Soriano, P., Llana-Belloch, S., \& Martinez-Patiño, M.J., (2013). Handgrip strength and hand dimensions in high-level interuniversity judoists. Archives of Budo, 9(1), 21-28.

Dempsey, P.G., \& Ayoub, M.M. (1996). The influence of gender, grasp type, pinch width and wrist position on sustained pinch strength. International Journal of Industrial Ergonomics, 17, 259273.

Dopsaj, M., Koropanovski, N., Vuckovic, G., Blagojevic, M., Marincovic B., \& Miljus, D. (2007). Maximal isometric handgrip force in well-trained university students in Serbia: descriptive, functional and sexual dimorphic model. Serbian Journal of Sports Sciences, 1, 139-148.

Farmosi, I. (1980). Body composition, somatotype and some motor performance of judoists. Journal of Sports Medicine and Physical Fitness, 20, 431-434.

Franchini, E., Del Vecchio, F.B., Matsushigue, K.A., \& Artioli, G. (2011). Physiological profiles of elite judo athletes. Sports Medicine, 41, 147-166.

Franchini, E., Nunes, A.V., Moraes, J.M., \& Del Vecchio, F.B. (2007). Physical fitness and anthropometrical profile of the Brazilian male judo team. Journal of Physiological Anthropology, 26, 59-67.

Franchini, E., Souza, C.E., Urasaki, R., Oliveira, R., Sauressig, F., \& Matheus, L. (2004). Teste de resistência de força isométrica e dinâmica na barra com o judogi. En: CD-Rom del III Congress of the Spanish Association of Sports Science. Valencia: Gráficas Mari Montañana.

Franchini, E., Takito, M.Y., \& Bertuzzi, R.C.M. (2005a). Morphological, physiological and technical variables in high-level college judoists. Archives of Budo, 1(1), 1-7.

Franchini, E., Takito, M.Y., \& Kiss, M.A. (2000). Somatótipo, composição corporal e força isométrica em diferentes períodos do treinamento em atletas de judô juvenis. Revista Treinamento Desportivo, 5(2), 4-10.

Franchini, E., Takito, M.Y., Kiss, M.A., \& Sterkowicz, S. (2005b). Physical fitness and anthropometrical differences between elite and non-elite judo players. Biology of Sport, 22, 315-328.

Franchini, E., Takito, M.Y., Matheus, L., Brito-Vieira, D.E., \& Kiss, M.A. (1997). Composição corporal, somatótipo e força isométrica em atletas da seleção brasileira universitária de judô. Âmbito Medicina Esportiva, 3(3), 21-29.

Fry, A.C., Ciroslan, D., Fry, M.D., LeRoux, C.D., Schilling, B.K., \& Chiu, L.Z. (2006). Anthropometric and performance variables discriminating elite American junior men weightlifters. Journal of Strength and Conditioning Research, 20(4), 861-866.

Gutiérrez-Sánchez, A., Soria-Domínguez, A., Pérez-Turpin, J.A, Cortell-Tormo, J.M., \& Suárez-Llorca, C. (2011). Importance of hand-grip strength as an indicator for predicting the results of competitions of young judokas. Archives of Budo, 7(3), 167-172.

Iglesias, E., Clavel, I., Dopico, J., \&Tuimil, J.L. (2003). Acute effect of the specific effort of judo on different strength manifestations and their relation with the reached cardiac frequency 
during

the

confrontation.

Rendimientodeportivo.com,

http://www.rendimientodeportivo.com/N006/Artic027.htm. Acceso 13 Abril 2004.

Imrhan, S.N. (2003). Two-handed static grip strengths in males: the influence of grip width. International Journal of Industrial Ergonomics, 31, 303-311.

Karlsson, J., Bonde-Petersen, F., Henriksson, J, \&Knuttgen, H.G. (1975). Effects of previous exercise with arms or legs on metabolism and performance in exhaustive exercise. Journal of Applied Physiology, 38(5), 763-767.

Leyk, D., Gorges, W., Ridder, D., Wunderlich, M., Ruther, T., Sievert, A., Essfeld, D. (2007). Hand-grip strength of young men, women and highly trained female athletes. European Journal of Applied Physiology, 99, 415-421.

Little, N.G. (1991). Physical performance attributes of junior and senior women, juvenile, junior and senior men judokas. Journal of Sports Medicine and Physical Fitness, 31, 510-520.

Marcon, G., Franchini, E., Vieira, D.E., \& Barros, T.L. (2007). Time structure and activities performed during a judo match. En: Del Vecchio FB, Franchini E (eds.) Annals of the $5^{\text {th }}$ International Judo Federation World Research Symposium. International Judo Federation, p. 49.

Marcon, G., Franchini, E., Jardim, J.R., \& Neto, T. (2010). Structural Analysis of Action and Time in Sports: Judo. Journal of Quantitative Analysis in Sports, 6(4), 1226.

Matsumoto, Y., Ogawa, S., Asami, T., et al. (1972). A follow-up study of the physical fitness of judoists (report I and II). Bulletin of the Association for the Scientific Studies on Judo, 4, 1-26.

Miarka, B., Julio, U., Del Vecchio, F.B., Calmet, M., Franchini, E. (2010). Técnica y táctica en judo: una revisión. Revista de Artes Marciales Asiáticas. 5(1), 91-112.

Miller, A.E., Macdougall, J.D., Tarnopolsky, M.A., \& Sale, D. (1993). Gender differences in strength and muscle fiber characteristics. European Journal of Applied Physiology, 66(3), 254-262.

Nevill, A.M., \& Holder, R.L. (2000). Modeling handgrip strength in the presence of confounding variables: results from the Allied Dunbar National Fitness Survey. Ergonomics, 43, 15471558.

Nicolay, C.W., \& Walker, A.L. (2005). Grip strength and endurance: influences of anthropometric variation, hand dominance, and gender. International Journal of Industrial Ergonomics, 35, 605-618.

Peebles, L., \& Norris, B. (2003). Filling 'gaps' in strength data for design. Applied Ergonomics, 34, 7388.

Peolsson, A., Hedlund, R., \& Oberg, B. (2001) Intra- and inter-tester reliability and reference values for hand strength. Journal of Rehabilitation Medicine, 33, 36-41.

Ruiz-Ruiz, J., Mesa, J.L., Gutiérrez, A., \& Castillo, M.J. (2002). Hand size influences optimal grip span in women but not in men. Journal of Hand Surgery, 27, 897-901.

Sahlin, K, (1978). Intracellular $\mathrm{pH}$ and energy metabolism in skeletal muscle of man. Acta Physiologica Scandinavica, 455, 1-56.

Schlüssel, M.M., Anjos, L.A., Vasconcellos, M.T., \& Kac, G. (2008). Reference values of handgrip dynamometry of healthy adults: a population-based study. Clinical Nutrition, 27, 601-607.

Silva, A.M., Fields, D.A., Heimsfields, S.A. et al. (2011). Relationship between changes in total-body water and fluid distribution with maximal forearm strength in elite judo athletes. Journal of Strength and Conditional Research, 25(9), 2488-2496.

Taylor, A.W., \& Brassard, L. (1981). A physiological profile of Canadian judo team. Journal of Sports Medicine and Physical Fitness, 21, 160-164.

Thomas, S.G., Cox, M.H., LeGal, Y.M., Verde, T.J., \& Smith, H.K. (1989). Physiological profiles of the Canadian national judo team. Canadian Journal of Sport Science, 14,142-147.

Vianna, L.C., Oliveira, R.B., \& Araújo, C.G. (2007). Age-related decline in handgrip strength differs according to gender. Journal of Strength and Conditional Research, 21(4), 1310-1314.

Visnapuu, M., \& Jürimäe, T. (2007). Handgrip strength and hand dimensions in young handball and basketball players. Journal of Strength and Conditional Research, 21(3), 923-929.

Walker, S., Siddiqi, T., \& Amundsen, L. (2002). Measurement of hand grip fatigue. In: Proceedings of the Annual Conference of the American Physical Therapy Association (APTA), APTA Journal Website. http://apps.apta.org/Custom/abstracts/pt2002/Abs02AuthIndex.cfm. Acceso 8 Abril 2012. 
Yamaji, S., Demura, S., Nagasawa, Y., \& Nakada, M. (2006). The influence of different target values and measurement times on the decreasing force curve during sustained static gripping work. Journal of Physiological Anthropology, 25, 23-28.

\section{Datos biográficos de los autores}

Juan G. Bonitch Góngora es profesor de la Facultad de Ciencias del Deporte de la Universidad de Granada desde 2007. Licenciado en Ciencias del Deporte por la Universidad de Granada. Doctor en Ciencias del Deporte por la Universidad de Granada. Investigador del grupo SEJ438 "Análisis y Control del Rendimiento Deportivo" (ACSP), cuenta en su haber con varias publicaciones científicas en revistas especializadas del área de las Ciencias del Deporte e indexadas en el Journal Citation Reports (JCR). Ha sido campeón de España en todas las categorías de edad, incluyendo el Campeonato de España Absoluto en 2008. Es Director Técnico del Club Deportivo Budo y Presidente de la Asociación Granadina de Clubes de Judo. E-mail: juanbonitch@ugr.es, juanbonitch@hotmail.com.

Filipa Almeida ha sido profesora de la Facultad de Ciencias del Deporte de la Escuela Autónoma de Dirección de Empresas (EADE. Universidad de Gales-Reino Unido) en 2012-2013. Licenciada en Ciencias del Deporte por la Universidad de Granada. Máster en Profesorado de E.S.O., Bachillerato, F.P. y Enseñanza de Idiomas (Especialidad de Educación Física) por la Universidad de Granada. Investigadora del grupo SEJ438 “Análisis y Control del Rendimiento Deportivo" (ACSP). Atleta en activo, ha sido Campeona Nacional de Portugal en 5 ocasiones, incluyendo el Título de Campeona de Portugal Absoluta en 2012. Ha participado en diversos torneos internacionales del circuito mundial de judo como miembro de la Selección Portuguesa de Judo. Es miembro del equipo docente del del Club Deportivo Budo y Directora del Área de Enseñanza de la Asociación Granadina de Clubes de Judo. E-mail: lua_d_prata@hotmail.com. 